\title{
Encephalitis by dengue virus and other arboviruses
}

\author{
Encefalite pelo vírus da dengue e outros arbovírus
}

Paulo Pereira Christo

Santa Casa de Belo Horizonte, Departamento de Neurologia, Belo Horizonte MG, Brazil.

Correspondence:

Paulo Pereira Christo;

Avenida Professor Alfredo Balena,

189 / sala 1708; $30130-100$

Belo Horizonte MG, Brasil;

E-mail;ppc@gold.com.br

Conflict of interest:

There is no conflict of interest to

declare.

Received 14 May 2015

Accepted 21 May 2015

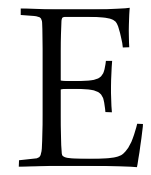

ncephalitis caused by arboviruses (ARthropod-BOrne viruses) is transmitted to humans through hematophagous arthropod bites. Emerging and re-emerging infections of arboviruses are a natural phenomenon associated with the evolution and adaptation of species and with complex factors, such as viral recombination and mutation, leading to more virulent and adapted strains, with urbanization and human activities that create a more permissive environment for vector-host interaction, and with increased air travel and trade ${ }^{1}$. The climate is also a determining factor in the temporal and geographical distribution of arthropods ${ }^{1}$. Brazil is a large tropical country and more than $1 / 3$ of its territory is covered by forests and other natural ecosystems and a diversity of flora and fauna that includes many arthropods, especially mosquitoes and midges ${ }^{2}$, thus making arboviruses an important public health problem.

Given the increase in international travel, arboviruses present in other countries, such as the West Nile Virus (WNV), Chikungunya and Zika virus, have already been introduced or will be introduced in the near future. Dengue outbreaks have been reported in most parts of Brazil in the last 15 years and there is serological evidence of other flavivirus infections, such as Saint Louis encephalitis virus (SLEV) and Rocio virus (ROCV), in horses bred in Brazil. These findings, coupled with the fact that positive serological tests for dengue may also show cross-reactivity with other flaviviruses, indicate the possibility that numerous cases of meningoencephalitis that occurred during recent dengue epidemics could have been incorrectly assigned to the disease ${ }^{3}$.

Northeastern, southeastern and southern Brazil are infested with Aedes aegypti, Aedes albopictus and Culex, which are important arbovirus vectors. More than 130 types of arbovirus can cause disease in humans and are responsible for a number of outbreaks of emerging infections in recent decades. The majority of arboviruses of importance to public health belong to three virus genera: Flavivirus (yellow fever virus, Japanese encephalitis virus, SLEV, WNV and ROCV; Alphavirus (Venezuelan equine encephalitis and Chikungunya); and Bunyavirus (Oropouche)1. In Brazil, three important Flavivirus and one Orthobunyavirus have been described as causes of infections of the central nervous system, ROCV, SLEV, dengue virus and Oropouche virus.

Dengue is caused by infection by one of the four serotypes of the dengue virus (DENV 1-4), is the most important emerging arbovirus in Brazil and worldwide and one of 17 neglected tropical diseases identified by the WHO that disproportionately affects the poorest populations the world 5 . In the last 50 years, its incidence has increased 30 -fold, with increasing geographic expansion to new countries and, in this decade, from urban to rural settings. Some 2.5 billion people are at risk, no less than $40 \%$ of the world's population in tropical and subtropical countries. An estimated 50 million dengue infections occur annually worldwide. Dengue is endemic in more than 100 countries in the regions of Africa, the Americas, the Eastern Mediterranean, South-East Asia and the Western Pacific 6 .

In Brazil, there are references to epidemics from the nineteenth century onward. The first clinical and laboratorial documentation of an epidemic occurred in 1981-82, in Boa Vista, Roraima, caused by serotypes 1 and 4. In 1986, there were epidemics in Rio de Janeiro and some capitals in the Northeast region. Since then, dengue has continuously occurred in Brazil, interspersed with the outbreak of epidemics, usually associated with the introduction of new serotypes into previously unaffected areas ${ }^{7}$. In 2013 , nearly 1.5 million cases were reported and from January to April 2015, about 800,000 cases have been recorded ${ }^{8}$. 
Population growth, uncontrolled urbanization in tropical and subtropical countries with poor sanitation, the proliferation of breeding foci for the Aedes mosquito and the lack of effective control of mosquitoes are potential reasons for the global resurgence and dissemination of dengue and other arboviruses9. Vector control campaigns for the dengue virus have not been successful and thus far, no vaccines have been approved for dengue. Vaccines have been under development since 1940 and there are now six vaccines in various stages of clinical trials ${ }^{10}$. The control of dengue and other arboviruses depends on a combination of factors, including early and accurate clinical and serological diagnosis, vaccination, environmental control that emphasizes a reduction in vector prevalence, reducing the susceptibility of the host and the development of specific and effective antivirals.

Clinical manifestations range from an asymptomatic state to severe dengue with plasma leakage, bleeding manifestations or organ impairment ${ }^{6}$. Classic dengue fever is characterized by the rapid onset of fever, headache, retro-orbital pain, myalgia, arthralgia, abdominal pain, nausea, vomiting and rash. Dengue fever can progress to more severe forms called dengue hemorrhagic fever and dengue shock syndrome. This traditional classification has been used for several years; however, it can be difficult to apply and numerous severe dengue cases have been lost in the midst of research ${ }^{11}$. In 2009, the WHO published new guidelines for defining dengue cases classified into three groups consisting of dengue fever without warning signs, dengue fever with warning signs and severe dengue (dengue with severe plasma leakage, severe bleeding or organ failure $)^{6}$. This classification also recognized the involvement of the CNS and alterations in the sensorium as criteria in the severity of dengue infection. However, specific neurological complications are not well described in the 2009 guidelines.

Neurological manifestations of DENV infections have been recorded in 0.5 to $20 \%$ of patients admitted to hospitals with classic dengue and 4 to $47 \%$ of patients admitted with encephalitis-like illness in endemic areas ${ }^{11}$.

A broad spectrum of neurological complications has been described. The revised and expanded edition implemented by the WHO Regional Office for South East Asia (SEARO) in 2011 classifies neurological manifestations as expanded dengue syndrome (unusual or atypical manifestations), which includes: febrile seizures in young children, encephalopathy, encephalitis, aseptic meningitis, intracranial hemorrhages/thrombosis, subdural effusions, mononeuropathies,
Guillain-Barré syndrome (GBS), transverse myelitis, myositis with elevated creatine phosphokinase (CPK), rhabdomyolysis, macular hemorrhage, impaired visual acuity, optic neuritis, post-infectious fatigue syndrome, depression, hallucinations and psychosis ${ }^{12}$. However these categories can overlap and clinical data like the cerebrospinal fluid examination (CSF) may be missing ${ }^{11}$.

Although diagnostic criteria for dengue encephalitis have been proposed, they are controversial, since the detection of dengue viral RNA and specific IgM antibodies in the CSF may be disease-course dependent and diagnostic tests are inconsistently available in endemic regions ${ }^{11,13}$.

The neurological complications of dengue virus infection can be classified based on the pathophysiology: disorders secondary to systemic complications, such as electrolyte disturbances, microcapillary hemorrhage, the release of toxic products and liver and kidney failure; post-infectious immune-mediated mechanisms, such as GBS and neuralgic amyotrophy; and the direct neurotrophic effect of the virus manifesting as encephalitis, myelitis and myositis ${ }^{14}$.

When patients present clinical syndromes of encephalitis, GBS and myelitis, infection by the dengue virus should always form part of the differential diagnosis, especially in endemic regions like Brazil or in people who have recently traveled to endemic regions.

Despite a recent increase in publications describing neurological complications in the course of infection by the dengue virus, the full extent of these complications and the pathophysiological mechanisms of these complications are not well established. Therefore, a review of the possible pathophysiological mechanisms of the disease is required. In this chapter, Puccioni-Sohler and Rosadas ${ }^{15}$ provide a clearer understanding of the possible pathophysiological mechanisms and seek to guide future research by improving compression of the gaps in the pathophysiology of the neurological involvement of dengue virus infection. The chapter discusses neuroinvasion, neurotropism, neurovirulence and animal models of infection with dengue virus in detail. While these issues remain elusive, new knowledge has emerged in recent years.

With increasing numbers of neurologists working in endemic regions and with the increase in globalization and international travel, the neurological manifestations of dengue must be more clearly recognized and understood to avoid being neglected. Neurologists must learn to diagnose, treat and assist in the elimination and eradication of dengue and other emerging arboviruses.
1. Dash AP, Bhatia R, Sunyoto T, Mourya DT. Emerging and re-emerging arboviral diseases in Southeast Asia.J Vector Borne Dis. 2013;50:77-84. 
3. Figueiredo LT. Saint Louis encephalitis virus and other arboviruses in the differential diagnosis for dengue. Rev Soc Bras Med Trop. 2007;47(5):541-2. http://dx.doi.org/10.1590/0037-8682-0197-2014

4. Bastos MS, Lessa N, Naveca FG, Monte RL, Braga WS, Figueiredo et al. Detection of Herpesvirus, Enterovirus, and Arbovirus Infection in Patients With Suspected Central Nervous System Viral Infection in the Western Brazilian Amazon. J Med Virol. 2014;86(9):1522-7. http://dx.doi.org/10.1002/jmv.23953

5. World Health Organization. Working to overcome the impact of neglected tropical diseases: first WHO report on neglected tropical diseases. Geneva: World Health Organization; 2010.

6. WHO. Dengue guidelines for diagnosis, treatment, prevention and control: new edition. Geneve: World Health Organization; 2009.

7. Ministério da Saúde (BR). Secretaria de Vigilância em Saúde. Guia de vigilância epidemiológica. 6a ed. Brasília, DF: Ministério da Saúde; 2005.

8. Monitoramento dos casos de dengue e febre de chikungunya até a semana epidemiológica 15, 2015. Bol Epidemiol. 2015;46(14).

9. Guzmán MG, Kouri G. Dengue: an update. Lancet Infect Dis. 2002;2(1):33-42. http://dx.doi.org/10.1016/S1473-3099(01)00171-2
10. Sinha G. Sanofi's dengue vaccine first to complete phase 3. Nat Biotechnol. 2014;32(7):605-6. http://dx.doi.org/10.1038/nbt0714-605a

11. Carod-Artal FJ, Wichmann O, Farrar J, Gascon J. Neurological complications of dengue virus infection. Lancet Neurol. 2013;12(9):906-19. http://dx.doi.org/10.1016/S1474-4422(13)70150-9

12. World Health Organization. Comprehensive guidelines for prevention and control of dengue and dengue haemorrhagic fever. Rev expand ed. Geneve: World Health Organization; 2011. (SEARO Technical publication series, vol 60).

13. Soares C, Puccioni-Sohler M. Diagnosis criteria of dengue encephalitis. Arq Neuropsiquiatr. 2013;72(3):263. http://dx.doi.org/10.1590/0004-282X20130251

14. Verma R, Sahu R, Holla V. Neurological manifestations of dengue infection: a review. J Neurol Sci. 2014;346(1-2):26-34. http://dx.doi.org/10.1016/j.jns.2014.08.044

15. Puccioni-Sohler M, Rosadas C. Advances and new insights in the neuropathogenesis of dengue infection. Arq Neuropsiquiatr. 2015;73(8):698-703. http://dx.doi.org/10.1590/0004-282X20150074 